\title{
JMASM30 PI-LCA: A SAS Program Computing the Two-point Mixture Index of Fit for Two-class LCA Models with Dichotomous Variables (SAS)
}

\author{
Dongquan Zhang \\ DMS International, dq.zhang@dmsinetwork.com \\ C. Mitchell Dayton \\ University of Maryland, cdayton@umd.edu
}

Follow this and additional works at: http://digitalcommons.wayne.edu/jmasm

Part of the Applied Statistics Commons, Social and Behavioral Sciences Commons, and the Statistical Theory Commons

\section{Recommended Citation}

Zhang, Dongquan and Dayton, C. Mitchell (2010) "JMASM30 PI-LCA: A SAS Program Computing the Two-point Mixture Index of Fit for Two-class LCA Models with Dichotomous Variables (SAS)," Journal of Modern Applied Statistical Methods: Vol. 9 : Iss. 1 , Article 32.

DOI: $10.22237 /$ jmasm/1272688260

Available at: http://digitalcommons.wayne.edu/jmasm/vol9/iss1/32

This Algorithms and Code is brought to you for free and open access by the Open Access Journals at DigitalCommons@WayneState. It has been accepted for inclusion in Journal of Modern Applied Statistical Methods by an authorized editor of DigitalCommons@WayneState. 


\section{JMASM ALGORITHMS AND CODE JMASM30 PI-LCA: A SAS Program Computing the Two-point Mixture Index of Fit for Two-class LCA Models with Dichotomous Variables (SAS)}

\author{
Dongquan Zhang \\ DMS International
}

\author{
C. Mitchell Dayton \\ University of Maryland \\ College Park
}

The two-point mixture index of fit enjoys some desirable features in model fit assessment and model selection, however, a need exists for efficient computational strategies. Applying an NLP algorithm, a program using the SAS matrix language is presented to estimate the two-point index of fit for two-class LCA models with dichotomous response variables. The program offers a tool to compute $\pi^{*}$ for twoclass models and it also provides an alternative program for conducting latent class analysis with SAS. This study builds a foundation for further research on computational approaches for M-class models.

Key words: Pi-star, two-class LCA models, SAS.

\section{Introduction}

The two-point mixture index of fit, $\pi^{*}$, was introduced to address the issue of model fit for frequency data in two-way contingency tables (Rudas, et al., 1994; Xi, 1994; Clogg, et al., 1995; Xi \& Lindsay, 1996). This index has been extended to a variety of other theoretical models. For example, Rudas \& Zwick (1997) discussed the use of $\pi^{*}$ in differential item functioning, Rudas (1999) studied applications of $\pi^{*}$ with regression models involving continuous variables and Dayton $(1999 ; 2003)$ extended the application of $\pi^{*}$ to latent class models.

$$
\text { For a two-point mixture, }
$$
$P=(1-\pi) \Phi+\pi \Psi$, let $\Phi$ denote the probability distribution of some hypothesized frequency model, $H$, let $\Psi$ represent an unspecified probability distribution, and let $\pi$ indicate the proportion of the population that is

Dongquan Zhang is an Operations Research Analyst in DMS International. Email: dq.zhang@dmsinetwork.com. C. Mitchell Dayton is a Professor Emeritus and past Chair in the Department of Measurement \& Statistics at the University of Maryland. Email: cdayton@umd.edu. not intrinsically described by model $H$. Then, the mathematical model for $\pi^{*}$ can be written as (Rudas, et al., 1994):

$$
\pi^{*}=\inf \{\pi \mid P=(1-\pi) \Phi+\pi \Psi, \Phi \in H\}
$$

In effect, $\pi^{*}$ is defined as the smallest value of $\pi$ for which $P$ remains true for model $H$ and can be viewed as "a measure of the proportion of the population measured with error" (Rudas, et al., 1994, p. 628) or as a measure of lack of fit (Rudas, et al., 1994; Xi, 1994; Xi \& Lindsay, 1996). In practice, the minimum proportion of cases that must be removed from the frequency table is compared to the remaining cases in order to provide perfect fit for $H$ (Dayton, 2003).

As opposed to conventional approaches, such as the $G^{2}$ likelihood ratio test and various information criteria such as AIC, $\pi^{*}$ represents a new perspective with respect to model-fit assessment and provides an easy-to-interpret alternative basis for model comparison and selection. Rudas, et al. (1994) summarized the desirable properties of this new index as: (1) unique; (2) defined on the 0, 1 interval; (3) decreasing in magnitude for increasingly more complex models when comparing nested models; and (4) invariant to multiplicative transformation of the frequency data. This latter 


\section{ZHANG \& DAYTON}

property is particularly interesting because it means that the magnitude of $\pi^{*}$ is not dependent on sample (although its sampling error is).

\section{Application of $\pi^{*}$ to Latent Class Models}

A latent class model with $T$ classes is, from a mathematical point of view, a finite mixture of product-multinomial probability functions. Considering a four-variable model as an example, the unconditional probability for the response vector, $Y=\{i j k l\}$, can be defined as:

$$
P(Y)=\sum_{t=1}^{T} \tau_{t} P(Y \mid t)
$$

where $\tau_{t}$ is the proportion in latent class $t$, and $P(Y \mid t)$ is the product of the conditional response probabilities for the four variables corresponding to the response pattern $\{i j k l\}$, given membership in latent class $t$. The latent class model is subject to the restrictions that: (1) the latent class proportions sum to 1 ; (2) the conditional response probabilities, given latent class membership, sum to 1 for each variable; (3) the variables are conditionally independent within any given class (Lazarsfeld \& Henry, 1968; Goodman, 1974; Dayton, 1999; among others).

In latent class analysis, Chi-square goodness-of fit tests and information criteria are widely applied procedures for assessing model fit and for model selection. These methods are open to the criticisms that: (1) with small sample size or sparse data, the statistics do not asymptotically follow appropriate $\chi^{2}$ distributions; and (2) with large sample size, it is highly likely that the null hypothesis will be rejected for relatively trivial effects. Therefore, Chi-square tests may not be appropriate for model selection under those circumstances. For information criteria, such as $A I C$, it is not clear how much the effect of sample size persists when the penalty term is applied. In addition, information criteria cannot be used to assess model fit in an absolute sense insomuch as interpretation of magnitudes of information criteria per se is difficult (Rudas, et al., 1994).
For the $s^{\text {th }}$ response vector, the latent class model can be incorporated into the twopoint mixture model as follows (Dayton, 2003):

$$
P_{s}=(1-\pi) P\left(y_{s}\right)+\pi \Psi_{s}
$$

where $P\left(y_{s}\right)$ represents the probability distribution for the $s^{\text {th }}$ response vector or response pattern. $\pi^{*}$ is obtained as the minimum value of $\pi$ when the model holds true across all response vectors (Dayton, 2003). The definition of $\pi^{*}$ circumvents the drawbacks of Chi-square statistics, thus, the index enjoys some unique advantages in model selection.

\section{Methodology \\ Computational Approach \\ Programs for LCA such as LEM or SAS} PROC LCA (Lanza, et al., 2007) do not provide options for computing $\pi^{*}$. However, $\pi^{*}$ can be estimated using the iterative procedures proposed by Rudas, et al. (1994) and with MLE or nonlinear programming (NLP) algorithms (Xi, 1994; Xi \& Lindsay, 1996). Dayton (2003) discusses computational strategies for the fit index applied to latent class and IRT (Rasch) models and presents examples using Microsoft Excel Solver, a program that is based on a NLP algorithm. For latent class models, Dayton (2003) detailed a computational strategy in two stages: in the first stage, the NLP parameters are defined as $a_{i t}, b_{j t}, c_{k t}, d_{l t}$, etc. such that $\hat{n}_{s}=a_{i t} \times b_{j t} \times c_{k t} \times d_{l t} \times \cdots$. Given the nonlinear constraint that the total expected frequency is equal to the total observed frequency, conventional MLEs of the parameters for an unrestricted latent class model can be estimated by minimizing $G^{2}$ as the objective function.

In the second stage, more nonlinear constraints, which specify the relationship between the expected frequency and the observed frequency for each response vector, are applied in NLP. The objective function is then redefined as maximizing the total expected frequency (or, equivalently, minimizing $\pi$, which is a function of the expected frequencies). After convergence to some preset criterion, an estimate of $\pi^{*}$ is obtained (Dayton, 2003). 


\section{PI-LCA SAS PROGRAM FOR TWO-CLASS LCA DICHOTOMOUS VARIABLE MODELS}

Technically, simply applying the second stage alone generates an estimate of $\pi^{*}$. However, an associated problem, which is increasingly crucial when the number of parameters increases, is the selection of start values because good start values are critical to computational efficiency and accuracy. With inappropriate start values, the optimization procedure may fail to converge, may converge at a local optimum, or may encounter other unexpected difficulties.

Although it is possible to provide different sets of start values and to examine the results in a single stage, a more efficient approach is to first conduct a conventional unrestricted LCA analysis and then start from the resultant parameter estimates which are, in general, closer to the final NLP estimates than arbitrarily selected start values. Although start values still need to be selected for first stage optimization, one benefit of the two-stage approach is that the closer estimates of the parameters are secured with only one (not multiple) constraints, no matter how many parameters are in the model. Hence, in the second stage, computational efficiency is achieved with faster convergence since the number of NLP function calls is greatly reduced.

Two SAS NLP subroutines, NLPNMS and NLPQN, are available to implement nonlinear constraints. The NLPQN subroutine applies quasi-Newton optimization technique that involves computing first-order partial derivatives in the gradient vector or the Jacobian matrix. It is suitable for medium to moderately large problems (NLPQN, SAS 9.1 Documentation, 2007) that contain relatively large numbers of parameters; NLPNMS is suitable for smaller problems. For nonlinearly constrained optimization, the NLPQN subroutine applies a modification of Powell's (1978, 1982) Variable Metric Constrained WatchDog algorithm (NLPQN call, SAS 9.1 Documentation, 2007). PI-LCA implements s the NLPQN subroutine for optimization.

\section{SAS Program Description}

As the computation involves relatively complex matrix operations, the current version of the procedure is restricted to two-class LCA models with dichotomous response variables. The SAS program, PI-LCA, is designed to compute $\pi^{*}$ for models for varying numbers of variables. However, for large numbers of variables computational time may become excessive. Factors influencing the number of function calls include selection of start values, number of parameters, and data structure, such as the number of zero-frequency vectors.

The SAS program PI-LCA has four sections:

1. Macro variables. Specifically, the following quantities are labeled and input as macro variables:

a. Number of dichotomous variables;

b. Number of latent classes (set at 2 in current version of program);

c. Observed sample size;

d. Start values for the first stage optimization;

e. Input data file name and location.

In this area of the program, the user must make adjustments in accordance with the data under consideration.

2. Data input for computing the expected frequencies. The data file can be any format (such as ASCII) that is acceptable to SAS. As the NLP procedure involves nonlinear constraints with regard to each response vector, aggregated data by the response pattern must be used as input. Assume that the number of items is numvar (as suggested previously), there should be numvar+1 fields in the dataset, with the first numvar fields representing the response patterns (e.g., 1111 for 4 items) - the last field being the observed frequency. For ASCII data input, such as the text data generated by Microsoft Notepad, the fields should be space delimited; for example: 1111 freq. For each observation (response pattern), the first numvar fields can either be 1's and 2's or 0's and 1's (see Table 1).

3. The first stage of the optimization procedure. This stage computes 


\section{ZHANG \& DAYTON}

conventional, unrestricted two-class LCA parameters using the NLPQN algorithm. The objective function that is minimized is $G^{2}$, given the constraint that the total observed frequency and the total expected frequency are equal. In addition, boundary constraints are applied to ensure that all parameter estimates are non-negative. Because start values are randomly selected in this stage, detailed NLP options (items 48 in the option vector for NLPQN, which may vary from case to case) are specified to obtain accurate estimates. The options may increase the number of function calls and make the convergence slower, especially when there are large numbers of parameters. When the procedure converges, the start values for the second stage are obtained. It is suggested that distinct sets of start values for the first stage should be tried to ensure that a global optimum has been obtained.

4. The second stage of the optimization procedure. In this stage, both the objective function and nonlinear constraints are redefined. In most cases, the convergence is relatively fast as the start values are close to optimum. In general, items 4-8 in the option vector for NLPQN do not need to be changed from default values. At convergence, the estimate of $\pi^{*}$ is obtained.
Results: Exemplary Data

Example 1: Academic Cheating Data (Four Items)

Dayton (2003) used Microsoft ExcelSolver to compute $\pi^{*}$ for a two-class LCA model with frequency data for four dichotomous ( 2 = yes, have engaged in this cheating behavior, and $1=$ no, have not engaged in this cheating behavior) items from a survey concerned with academic cheating behavior by college students (see Table 1).

Table 1: Academic Cheating Data

\begin{tabular}{|c|c|c|c|c|}
\hline \multicolumn{4}{|c|}{ Item } & \multirow{2}{*}{ Frequency } \\
\hline A & B & $\mathrm{C}$ & D & \\
\hline 1 & 1 & 1 & 1 & 207 \\
\hline 1 & 1 & 1 & 2 & 46 \\
\hline 1 & 1 & 2 & 1 & 7 \\
\hline 1 & 1 & 2 & 2 & 5 \\
\hline 1 & 2 & 1 & 1 & 13 \\
\hline 1 & 2 & 1 & 2 & 4 \\
\hline 1 & 2 & 2 & 1 & 1 \\
\hline 1 & 2 & 2 & 2 & 2 \\
\hline 2 & 1 & 1 & 1 & 10 \\
\hline 2 & 1 & 1 & 2 & 3 \\
\hline 2 & 1 & 2 & 1 & 1 \\
\hline 2 & 1 & 2 & 2 & 2 \\
\hline 2 & 2 & 1 & 1 & 11 \\
\hline 2 & 2 & 1 & 2 & 4 \\
\hline 2 & 2 & 2 & 1 & 1 \\
\hline 2 & 2 & 2 & 2 & 2 \\
\hline & & & & 319 \\
\hline
\end{tabular}

Input to Section (1) of the SAS Program

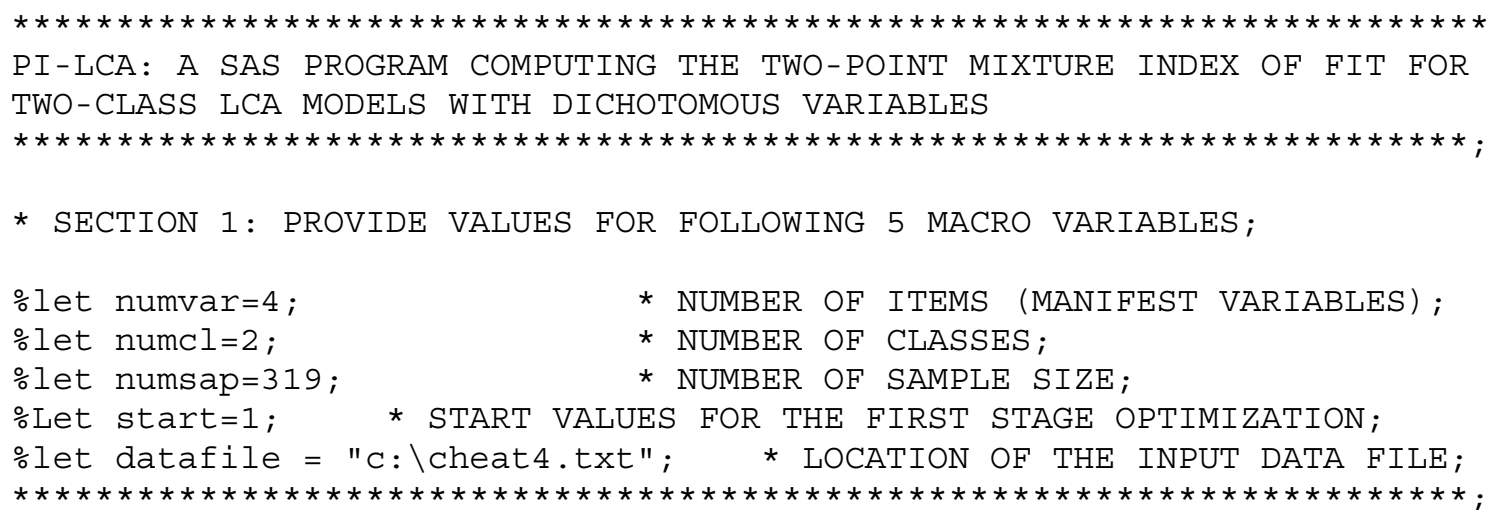




\section{PI-LCA SAS PROGRAM FOR TWO-CLASS LCA DICHOTOMOUS VARIABLE MODELS}

Selected Output: SAS output 1- Call NLPQN Subroutine in the First Stage to Conduct Latent Class Analysis Computing Pi-star, the Two-Point Mixture Fit Index

The First Stage: Latent Class Analysis

The Objective Function Is to Minimize G-square

Iterations

Optimization Results

Gradient Calls

98 Function Calls

107

Objective Function

100 Active Constraints

3.8821212398 Maximum Constraint $1.4590216 \mathrm{E}-7$

Violation

Maximum Projected Gradient 0.0009804183 Value Lagrange Function 3.8821210939

Maximum Gradient of the

0.0008997954 Slope of Search Direction

$-3.02492 \mathrm{E}-7$

Lagran Func

$\begin{array}{rl}N & \text { Parameter } \\ 1 & \text { X1 } \\ 2 & X 2 \\ 3 & X 3 \\ 4 & X 4 \\ 5 & X 5 \\ 6 & X 6 \\ 7 & X 7 \\ 8 & \text { X8 } \\ 9 & X 9 \\ 10 & X 10 \\ 11 & X 11 \\ 12 & X 12 \\ 13 & X 13 \\ 14 & X 14 \\ 15 & X 15 \\ 16 & X 16\end{array}$

Parameter Estimates

Gradient Gradient

Objective Lagrange

Estimate Function

$8.023430 \quad-32.820114 \quad 0.000030386$

$\begin{array}{lll}4.030813 & -64.491314 & 0.000059927\end{array}$

$2.585951 \quad-99.709946 \quad 0.000146$

$\begin{array}{lll}2.407726 & -90.980625 & 0.000146\end{array}$

$0.135555 \quad-32.819815 \quad 0.000329$

$0.121356 \quad-64.490988 \quad 0.000386$

$0.099626 \quad-99.710992 \quad-0.000900$

$\begin{array}{lll}0.535523 & -90.980890 & -0.000119\end{array}$

$\begin{array}{lll}1.361351 & -15.918088 & 0.000058771\end{array}$

$\begin{array}{lll}1.203668 & -17.486176 & 0.000071031\end{array}$

$1.237312 \quad-32.453413 \quad 0.000193$

$\begin{array}{lll}1.147240 & -14.876575 & 0.000191\end{array}$

$\begin{array}{lll}1.856461 & -15.917976 & 0.000171\end{array}$

$\begin{array}{lll}1.725583 & -17.486067 & 0.000181\end{array}$

$\begin{array}{lll}0.340991 & -32.453140 & 0.000466\end{array}$

$1.295821 \quad-14.876776-0.000010208$

Value of Objective Function $=3.8821212398$

Value of Lagrange Function $=3.8821210939$

Latent Class Analysis

Observed Frequency Expected Frequency

$207 \quad 205.71667$

$46 \quad 47.414163$

$7 \quad 8.9574477$

$5 \quad 2.4494936$

$13 \quad 12.303603$

5.1148321

1.9535633

1.0899392

9.3388155

4.3394233

1.7671869

1.0165214

8.6134428

5.1590278

2.3494963

1.4163751

Total Expected Frequency

319

LC1 Proportion LC2 Proportion

$$
0.839431 \quad 0.160569
$$

Conditional Probabilities

CP Positive Response (1) $\quad 10.9833858 \quad 0.4230674$

$2 \quad 0.970773 \quad 0.4109132$

$30.9629034 \quad 0.783951$

$\begin{array}{lll}4 & 0.8180504 & 0.6236428\end{array}$

CP Negative Response (2)

10.01661420 .5769326

$\begin{array}{lll}2 & 0.029227 & 0.5890868\end{array}$

$\begin{array}{lll}3 & 0.0370966 & 0.216049\end{array}$

$\begin{array}{lll}4 & 0.1819496 & 0.3763572\end{array}$ 


\section{ZHANG \& DAYTON}

SAS Output 2: Call NLPQN Subroutine in the Second Stage to Compute $\pi^{*}$

Computing Pi-star, the Two-Point Mixture Fit Index

The Second Stage: Pi Optimization

The Objective Function Is to Maximize the Total Expected Frequency

Iterations
Gradient Calls
Objective Function
Maximum Projected Gradien
Maximum Gradient of the
Lagran Func
\[ \]
\[ \begin{array}{ll}\text { N Parameter } \\ 1 \times 1 \\ 2 \times 2 \\ 3 \times 3 \\ 4 \times 4 \\ 5 \times 5 \\ 6 \times 6 \\ 7 \times 7 \\ 8 \times 8 \\ 9 \times 9 \\ 10 \times 10 \\ 11 \times 11 \\ 12 \times 12 \\ 13 \times 13 \\ 14 \times 14 \\ 15 \times 15 \\ 16 \times 16\end{array} \]

Optimization Results

6 Function Calls $\quad 8$

$\begin{array}{rlr}8 & \text { Active Constraints } & 10 \\ 310.01091238 & \text { Maximum Constraint } & 9.3996391 \mathrm{E}-8\end{array}$

3.9912676E-7 Value Lagrange Function $\quad-310.0109122$

3.0088341E-7 Slope of Search Direction $-4.425832 \mathrm{E}-7$

Parameter Estimates

\begin{tabular}{lrr}
\multicolumn{2}{c}{ Gradient Objective } & Gradient Lagrange \\
Estimate & Function & Function \\
8.033189 & 32.624665 & $2.9195017 \mathrm{E}-9$ \\
4.037358 & 64.709589 & -0.000000232 \\
2.592207 & 100.059213 & -0.000000175 \\
2.414567 & 90.971362 & 0.000000301 \\
0.176867 & 32.624664 & $6.428263 \mathrm{E}-11$ \\
0.101910 & 64.709588 & $-5.847028 \mathrm{E}-9$ \\
0.084711 & 100.059213 & $-5.726554 \mathrm{E}-9$ \\
0.529770 & 90.971362 & $6.6015519 \mathrm{E}-8$ \\
1.324498 & 13.361448 & $-4.779081 \mathrm{E}-8$ \\
0.937841 & 15.143763 & $7.451175 \mathrm{E}-8$ \\
1.350402 & 28.603220 & $-8.034978 \mathrm{E}-8$ \\
2.385143 & 12.948459 & $3.8478315 \mathrm{E}-8$ \\
1.830894 & 13.361447 & $-6.606271 \mathrm{E}-8$ \\
1.846183 & 15.143764 & 0.000000147 \\
0.123579 & 28.603222 & $-7.353005 \mathrm{E}-9$ \\
0.870889 & 12.948459 & $1.4049627 \mathrm{E}-8$
\end{tabular}

Value of Objective Function $=310.01091238$

Value of Lagrange Function $=310.01091224$

Pi-Star Results

Observed Frequency Expected Frequency

$\begin{array}{rc}207 & 207 \\ 46 & 46 \\ 7 & 7 \\ 5 & 1.5891942 \\ 13 & 13 \\ 4 & 4 \\ 1 & 0.8881988 \\ 2 & 0.2999071 \\ 10 & 10 \\ 3 & 3 \\ 1 & 0.6521739 \\ 2 & 0.2168445 \\ 11 & 11 \\ 4 & 4 \\ 1 & 1 \\ 2 & 0.3645937\end{array}$

Total Expected Frequency: 310.01091

Pi-Star: 0.028179

LC1 Proportion LC2 Proportion

$0.8640029 \quad 0.1359971$

Conditional Probabilities

$\begin{array}{lllr}\text { CP Positive Response (1) } & 1 & 0.9784573 & 0.419757 \\ & 2 & 0.9753798 & 0.3368651 \\ & 3 & 0.9683548 & 0.9161599 \\ & 4 & 0.8200717 & 0.7325305 \\ & & & \\ & 1 & 0.0215427 & 0.580243 \\ \text { CP Negative Response (2) } & 2 & 0.0246202 & 0.6631349 \\ & 3 & 0.0316452 & 0.0838401 \\ & 4 & 0.1799283 & 0.2674695\end{array}$




\section{PI-LCA SAS PROGRAM FOR TWO-CLASS LCA DICHOTOMOUS VARIABLE MODELS}

In this example, the start values for all the parameters are set equal to 1 . In general, distinct sets of start values should be employed to ensure a global maximum. In this stage, there are 98 iterations and 107 function calls. The maximum constraint violation is in the range of 1E-6, which is acceptable. The objective function $\left(G^{2}\right)$ is minimized at 3.88. With the NLP parameters, the latent class proportions and the conditional probabilities $(\mathrm{CP})$ for the LCA model are computed.

The start values are imported from the first stage output. The objective function is redefined as maximizing the total expected frequency, which converges at 310.01 (in contrast to the total observed frequency of 319). There are only 6 iterations and 8 function calls prior to convergence (compared to 98 and 107 in the first stage). The estimated value of $\pi^{*}$ converges at 0.028 . Thus, only $2.8 \%$ of the cases in the population are estimated as not described by the two-class model; this suggests adequate model-data fit. (See SAS Output 2.)

Example 2: Drug Use Data (Five Items)

Five dichotomous $(2=$ yes, have used this drug and $1=$ no, have not used this drug) items in the drug use data set with a large number of zero frequencies (see Table 2). Following the approach of Clogg, et al. (1991) in applying flattening constants to deal with the sparse data that do not support conventional maximum likelihood analysis, zero frequencies are replaced with 0.5 , which enables the NLP optimization to converge. This increased the total frequency from 7,224 to 7,233.

Table 2: Drug Use Data

\begin{tabular}{|c|c|c|c|c|c|c|}
\hline \multicolumn{5}{|c|}{ Item } & \multicolumn{2}{c|}{ Frequency } \\
\hline A & B & C & D & E & Original & Replaced \\
\hline 1 & 1 & 1 & 1 & 1 & 710 & 710 \\
\hline 1 & 1 & 1 & 1 & 2 & 0 & 0.5 \\
\hline 1 & 1 & 1 & 2 & 1 & 0 & 0.5 \\
\hline 1 & 1 & 1 & 2 & 2 & 0 & 0.5 \\
\hline 1 & 1 & 2 & 1 & 1 & 4 & 4 \\
\hline 1 & 1 & 2 & 1 & 2 & 0 & 0.5 \\
\hline 1 & 1 & 2 & 2 & 1 & 0 & 0.5 \\
\hline 1 & 1 & 2 & 2 & 2 & 0 & 0.5 \\
\hline 1 & 2 & 1 & 1 & 1 & 263 & 263 \\
\hline 1 & 2 & 1 & 1 & 2 & 0 & 0.5 \\
\hline 1 & 2 & 1 & 2 & 1 & 0 & 0.5 \\
\hline 1 & 2 & 1 & 2 & 2 & 0 & 0.5 \\
\hline 1 & 2 & 2 & 1 & 1 & 21 & 21 \\
\hline 1 & 2 & 2 & 1 & 2 & 0 & 0.5 \\
\hline 1 & 2 & 2 & 2 & 1 & 0 & 0.5 \\
\hline 1 & 2 & 2 & 2 & 2 & 0 & 0.5 \\
\hline
\end{tabular}

Data continues in next table

\begin{tabular}{|c|c|c|c|c|c|c|}
\hline \multicolumn{5}{|c|}{ Item } & \multicolumn{2}{c|}{ Frequency } \\
\hline A & B & C & D & E & Original & Replaced \\
\hline 2 & 1 & 1 & 1 & 1 & 882 & 882 \\
\hline 2 & 1 & 1 & 1 & 2 & 0 & 0.5 \\
\hline 2 & 1 & 1 & 2 & 1 & 5 & 5 \\
\hline 2 & 1 & 1 & 2 & 2 & 0 & 0.5 \\
\hline 2 & 1 & 2 & 1 & 1 & 168 & 168 \\
\hline 2 & 1 & 2 & 1 & 2 & 0 & 0.5 \\
\hline 2 & 1 & 2 & 2 & 1 & 33 & 33 \\
\hline 2 & 1 & 2 & 2 & 2 & 0 & 0.5 \\
\hline 2 & 2 & 1 & 1 & 1 & 2636 & 2636 \\
\hline 2 & 2 & 1 & 1 & 2 & 0 & 0.5 \\
\hline 2 & 2 & 1 & 2 & 1 & 5 & 5 \\
\hline 2 & 2 & 1 & 2 & 2 & 0 & 0.5 \\
\hline 2 & 2 & 2 & 1 & 1 & 1716 & 1716 \\
\hline 2 & 2 & 2 & 1 & 2 & 17 & 17 \\
\hline 2 & 2 & 2 & 2 & 1 & 668 & 668 \\
\hline 2 & 2 & 2 & 2 & 2 & 96 & 96 \\
\hline & & & & & & \\
\hline
\end{tabular}




\title{
ZHANG \& DAYTON
}

\author{
Input to Section (1)
}

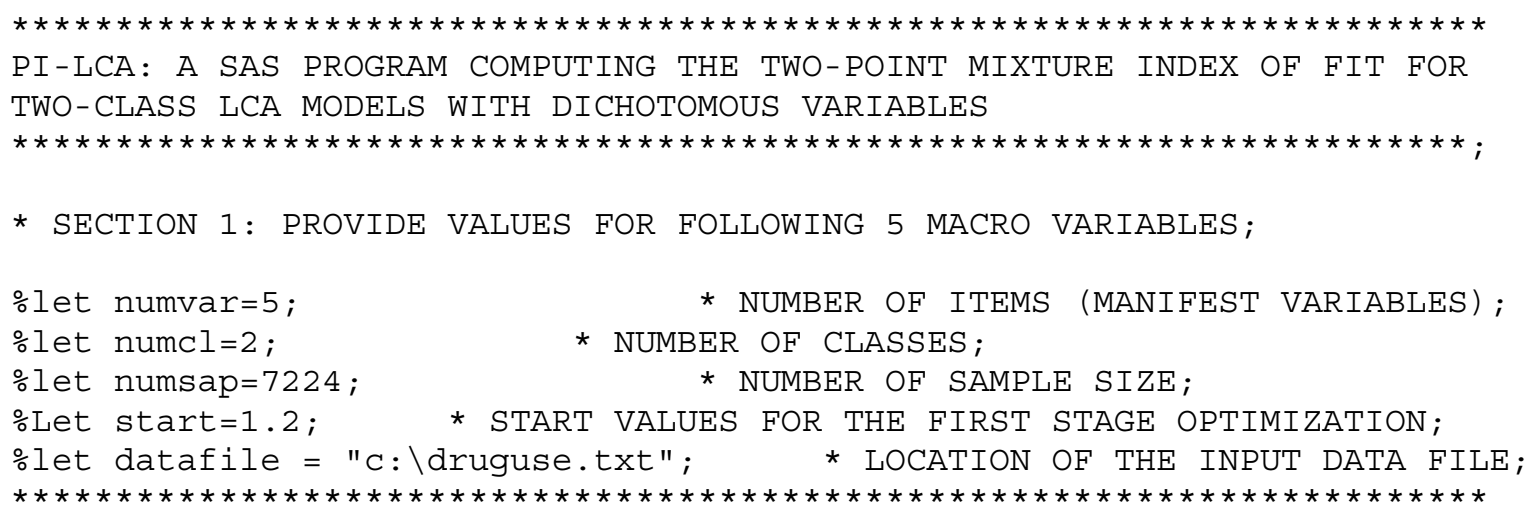

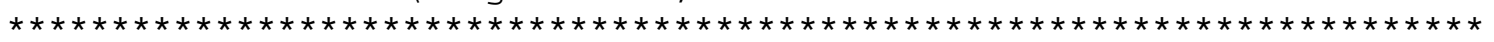

* NUMBER OF ITEMS (MANIFEST VARIABLES);

* NUMBER OF CLASSES;

* NUMBER OF SAMPLE SIZE;

* START VATUES FOR THE

SAS output 1- Call NLPQN Subroutine in the First Stage to Conduct Latent Class Analysis

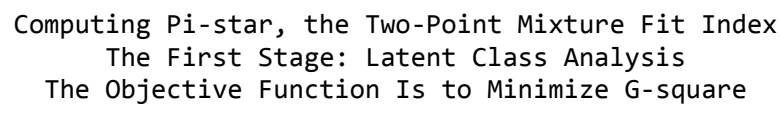

Iterations Gradient Calls Objective Function

Maximum Projected Gradient Maximum Gradient of the Lagran Func

\author{
Latent Class Analysis \\ Total Expected Frequency \\ 7233 \\ LC1 Proportion LC2 Proportion \\ $0.6394273 \quad 0.3605727$ \\ Conditional Probabilities \\ CP Positive Response (1) \\ $\begin{array}{llll} & 0.2155591 & 0.0027008\end{array}$ \\ 20.35615290 .0612726 \\ $\begin{array}{llll}3 & 0.9700971 & 0.0074087\end{array}$ \\ $\begin{array}{llll}4 & 0.9981559 & 0.6917313\end{array}$ \\ $\begin{array}{llll}5 & 0.9994005 & 0.9550512\end{array}$ \\ CP Negative Response (2) \\ $\begin{array}{llll}1 & 0.7844409 & 0.9972992\end{array}$ \\ $\begin{array}{lllll}2 & 0.6438471 & 0.9387274\end{array}$ \\ $\begin{array}{lll}3 & 0.0299029 & 0.9925913\end{array}$ \\ $\begin{array}{llll}4 & 0.0018441 & 0.3082687\end{array}$ \\ $\begin{array}{lllll}5 & 0.0005995 & 0.0449488\end{array}$
}




\section{ZHANG \& DAYTON}

Table 3: Abortion Data

\begin{tabular}{|c|c|c|c|c|c|c|c|c|c|c|c|c|c|}
\hline \multicolumn{6}{|c|}{ Item } & \multirow{2}{*}{ Frequency } & \multicolumn{6}{|c|}{ Item } & \multirow{2}{*}{ Frequency } \\
\hline A & $\mathrm{B}$ & $\mathrm{C}$ & $\mathrm{D}$ & $\mathrm{E}$ & $\mathrm{F}$ & & A & B & $\mathrm{C}$ & D & $\mathrm{E}$ & $\mathrm{F}$ & \\
\hline 1 & 1 & 1 & 1 & 1 & 1 & 10728 & 2 & 1 & 1 & 1 & 1 & 1 & 61 \\
\hline 1 & 1 & 1 & 1 & 1 & 2 & 732 & 2 & 1 & 1 & 1 & 1 & 2 & 24 \\
\hline 1 & 1 & 1 & 1 & 2 & 1 & 12 & 2 & 1 & 1 & 1 & 2 & 1 & 2 \\
\hline 1 & 1 & 1 & 1 & 2 & 2 & 24 & 2 & 1 & 1 & 1 & 2 & 2 & 6 \\
\hline 1 & 1 & 1 & 2 & 1 & 1 & 413 & 2 & 1 & 1 & 2 & 1 & 1 & 7 \\
\hline 1 & 1 & 1 & 2 & 1 & 2 & 503 & 2 & 1 & 1 & 2 & 1 & 2 & 25 \\
\hline 1 & 1 & 1 & 2 & 2 & 1 & 7 & 2 & 1 & 1 & 2 & 2 & 1 & 5 \\
\hline 1 & 1 & 1 & 2 & 2 & 2 & 53 & 2 & 1 & 1 & 2 & 2 & 2 & 11 \\
\hline 1 & 1 & 2 & 1 & 1 & 1 & 29 & 2 & 1 & 2 & 1 & 1 & 1 & 15 \\
\hline 1 & 1 & 2 & 1 & 1 & 2 & 11 & 2 & 1 & 2 & 1 & 1 & 2 & 7 \\
\hline 1 & 1 & 2 & 1 & 2 & 1 & 1 & 2 & 1 & 2 & 1 & 2 & 1 & 0 \\
\hline 1 & 1 & 2 & 1 & 2 & 2 & 1 & 2 & 1 & 2 & 1 & 2 & 2 & 9 \\
\hline 1 & 1 & 2 & 2 & 1 & 1 & 7 & 2 & 1 & 2 & 2 & 1 & 1 & 6 \\
\hline 1 & 1 & 2 & 2 & 1 & 2 & 9 & 2 & 1 & 2 & 2 & 1 & 2 & 7 \\
\hline 1 & 1 & 2 & 2 & 2 & 1 & 4 & 2 & 1 & 2 & 2 & 2 & 1 & 2 \\
\hline 1 & 1 & 2 & 2 & 2 & 2 & 3 & 2 & 1 & 2 & 2 & 2 & 2 & 12 \\
\hline 1 & 2 & 1 & 1 & 1 & 1 & 774 & 2 & 2 & 1 & 1 & 1 & 1 & 48 \\
\hline 1 & 2 & 1 & 1 & 1 & 2 & 1059 & 2 & 2 & 1 & 1 & 1 & 2 & 91 \\
\hline 1 & 2 & 1 & 1 & 2 & 1 & 18 & 2 & 2 & 1 & 1 & 2 & 1 & 4 \\
\hline 1 & 2 & 1 & 1 & 2 & 2 & 60 & 2 & 2 & 1 & 1 & 2 & 2 & 34 \\
\hline 1 & 2 & 1 & 2 & 1 & 1 & 641 & 2 & 2 & 1 & 2 & 1 & 1 & 46 \\
\hline 1 & 2 & 1 & 2 & 1 & 2 & 5643 & 2 & 2 & 1 & 2 & 1 & 2 & 1100 \\
\hline 1 & 2 & 1 & 2 & 2 & 1 & 21 & 2 & 2 & 1 & 2 & 2 & 1 & 3 \\
\hline 1 & 2 & 1 & 2 & 2 & 2 & 1181 & 2 & 2 & 1 & 2 & 2 & 2 & 1040 \\
\hline 1 & 2 & 2 & 1 & 1 & 1 & 7 & 2 & 2 & 2 & 1 & 1 & 1 & 6 \\
\hline 1 & 2 & 2 & 1 & 1 & 2 & 14 & 2 & 2 & 2 & 1 & 1 & 2 & 8 \\
\hline 1 & 2 & 2 & 1 & 2 & 1 & 1 & 2 & 2 & 2 & 1 & 2 & 1 & 3 \\
\hline 1 & 2 & 2 & 1 & 2 & 2 & 3 & 2 & 2 & 2 & 1 & 2 & 2 & 6 \\
\hline 1 & 2 & 2 & 2 & 1 & 1 & 10 & 2 & 2 & 2 & 2 & 1 & 1 & 8 \\
\hline 1 & 2 & 2 & 2 & 1 & 2 & 153 & 2 & 2 & 2 & 2 & 1 & 2 & 264 \\
\hline 1 & 2 & 2 & 2 & 2 & 1 & 2 & 2 & 2 & 2 & 2 & 2 & 1 & 1 \\
\hline 1 & 2 & 2 & 2 & 2 & 2 & 121 & 2 & 2 & 2 & 2 & 2 & 2 & 2045 \\
\hline
\end{tabular}

Total: $\quad 27,151$ 


\section{PI-LCA SAS PROGRAM FOR TWO-CLASS LCA DICHOTOMOUS VARIABLE MODELS}

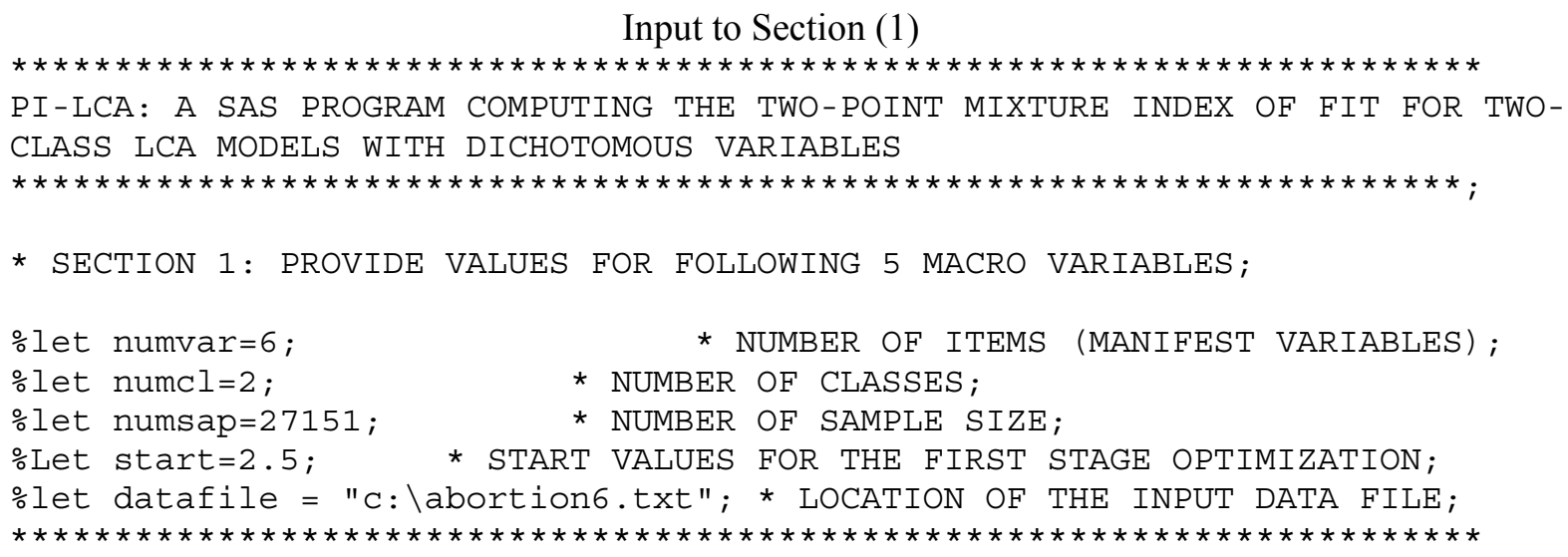

SAS output 1- Call NLPQN Subroutine in the First Stage to Conduct Latent Class Analysis

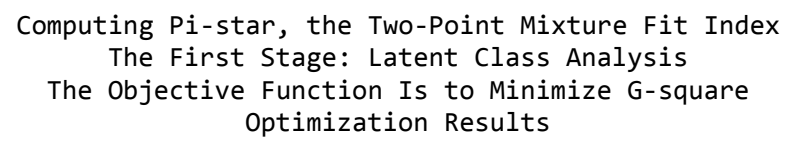

Iterations Gradient Calls

Objective Function
67 Function Calls

69 Active Constraints

5356.558615 Maximum Constraint Violation

Maximum Projected Gradient 8.5964821829 Value Lagrange Function Maximum Gradient of the Lagran Func
8.7492309364 Slope of Search Direction

Latent Class Analysis

Total Expected Frequency

27203.5

LC1 Proportion LC2 Proportion
0.4834715
0.5165285

Conditional Probabilities

CP Positive Response

(1)

$$
\begin{array}{lll}
1 & 0.9923362 & 0.6579907 \\
2 & 0.9209919 & 0.0480761 \\
3 & 0.9962743 & 0.8059632 \\
4 & 0.9546303 & 0.0921695 \\
5 & 0.9987107 & 0.6670409 \\
6 & 0.9257254 & 0.0547449
\end{array}
$$

CP Negative Response (2)
$10.0076638 \quad 0.3420093$
$\begin{array}{llll}2 & 0.0790081 & 0.9519239\end{array}$
$\begin{array}{llll}3 & 0.0037257 & 0.1940368\end{array}$
$\begin{array}{llll}4 & 0.0453697 & 0.9078305\end{array}$
$\begin{array}{llll}5 & 0.0012893 & 0.3329591\end{array}$
$\begin{array}{llll}6 & 0.0742746 & 0.9452551\end{array}$ 


\section{ZHANG \& DAYTON}

SAS output 2: Call NLPQN Subroutine in the Second Stage to Compute $\pi^{*}$

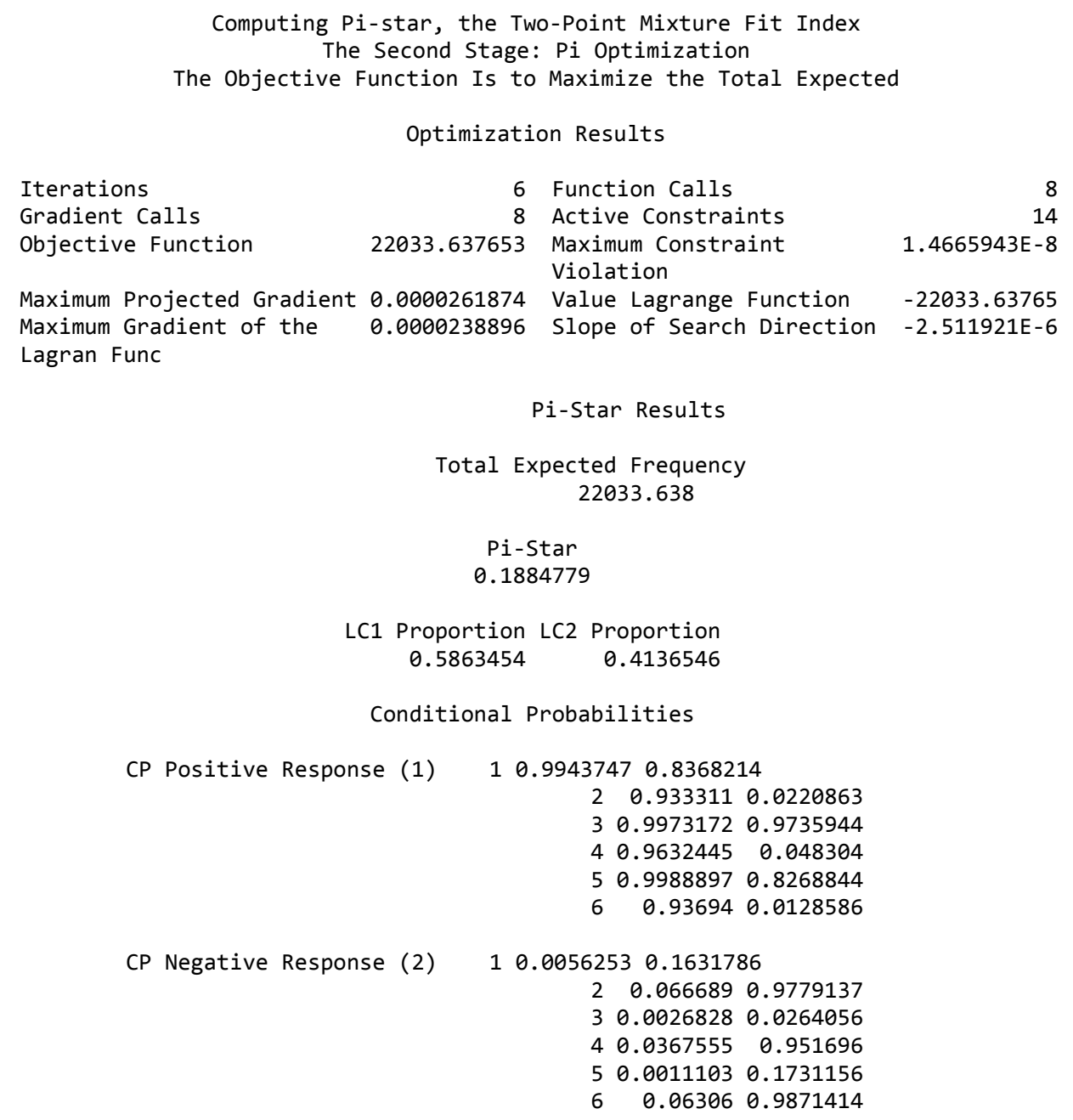

A vector of values equal to 2.5 was selected as start values. While the latent class proportions are $58 \%$ and $42 \%$, respectively, the value of $\pi^{*}$ is near 0.188 , indicating that in order to provide perfect fit, around $19 \%$ of the cases in the population are not taken into account. This suggests that the two-class model does not provide adequate fit; Dayton (2006) considered more complex models for these data.

\section{References}

Clogg, C. C., Rudas, T., \& Xi, L. (1995). A new index of structure for the analysis of models for mobility tables and other crossclassification. In P. Marsden (Ed.), Sociological Methodology, 197-222. Oxford. Blackwell.
Clogg, C. C., Rubin, D. B., Schenker, N., Schultz, B., \& Weidman, L. (1991). Multiple imputation of industry and occupation codes in census public-use samples using Bayesian logistic regression. Journal of the American Statistical Association, 86(413), 68-78.

Dayton, C. M. (1999). Latent class scaling analysis. Thousand Oaks, CA: Sage.

Dayton, C. M. (2003). Applications and Computational Strategies for the two-point mixture index of fit. British Journal of Mathematical and Statistical Psychology, 56, 113.

Dayton, C. M. (2006). Latent structure of attitudes toward abortion. In Real Data Analysis, S. S. Sawilowsky (Ed), AERA SIG/ES, 293-298. 


\section{PI-LCA SAS PROGRAM FOR TWO-CLASS LCA DICHOTOMOUS VARIABLE MODELS}

Goodman, L. A. (1974). Exploratory latent structure analysis using both identifiable and unidentifiable models. Biometrika, 61, 215231.

Lanza, S. T., Lemmon, D. R., Schafer, J. L.,\& Collins, L. M. (2007). PROC LCA \& PROC LTA user's guide Version 1.13 Beta. The Methodology Center, Pennsylvania State University.

Lazarsfeld, P. F., \& Henry, N. W. (1968). Latent structure analysis. Boston: Houghton Mifflin Company.

Rudas, T. (1999). The mixture index of fit and minimax regression. Metrika, 50, 163172.

Rudas, T., Clogg, C. C., \& Lindsay, B. G. (1994). A new index of fit based on mixture methods for the analysis of contingency tables. Journal of the Royal Statistical Society, Series B, 56, 623-639.
Rudas, T., \& Zwick, R. (1997). Estimating the importance of differential item functioning. Journal of Educational and Behavioral Statistics, 22, 31-45.

SAS. (2007). SAS OnlineDoc 9.1.3. SAS Institute, Inc. Retrieved June 10, 2007, from

http://support.sas.com/onlinedoc/913/docMainpa ge.jsp.

$\mathrm{Xi}, \mathrm{L} .(1994)$. The mixture index of fit for the independence model in contingency tables. Master of Arts paper, Department of Statistics, Pennsylvania State University.

Xi, L., \& Lindsay, B. G. (1996). A note on calculating the $\pi^{*}$ index of fit for the analysis of contingency tables. Sociological Methods \& Research, 25, 248-259.

Appendix: The SAS Program to Compute Pi-star with the Cheat4 Data

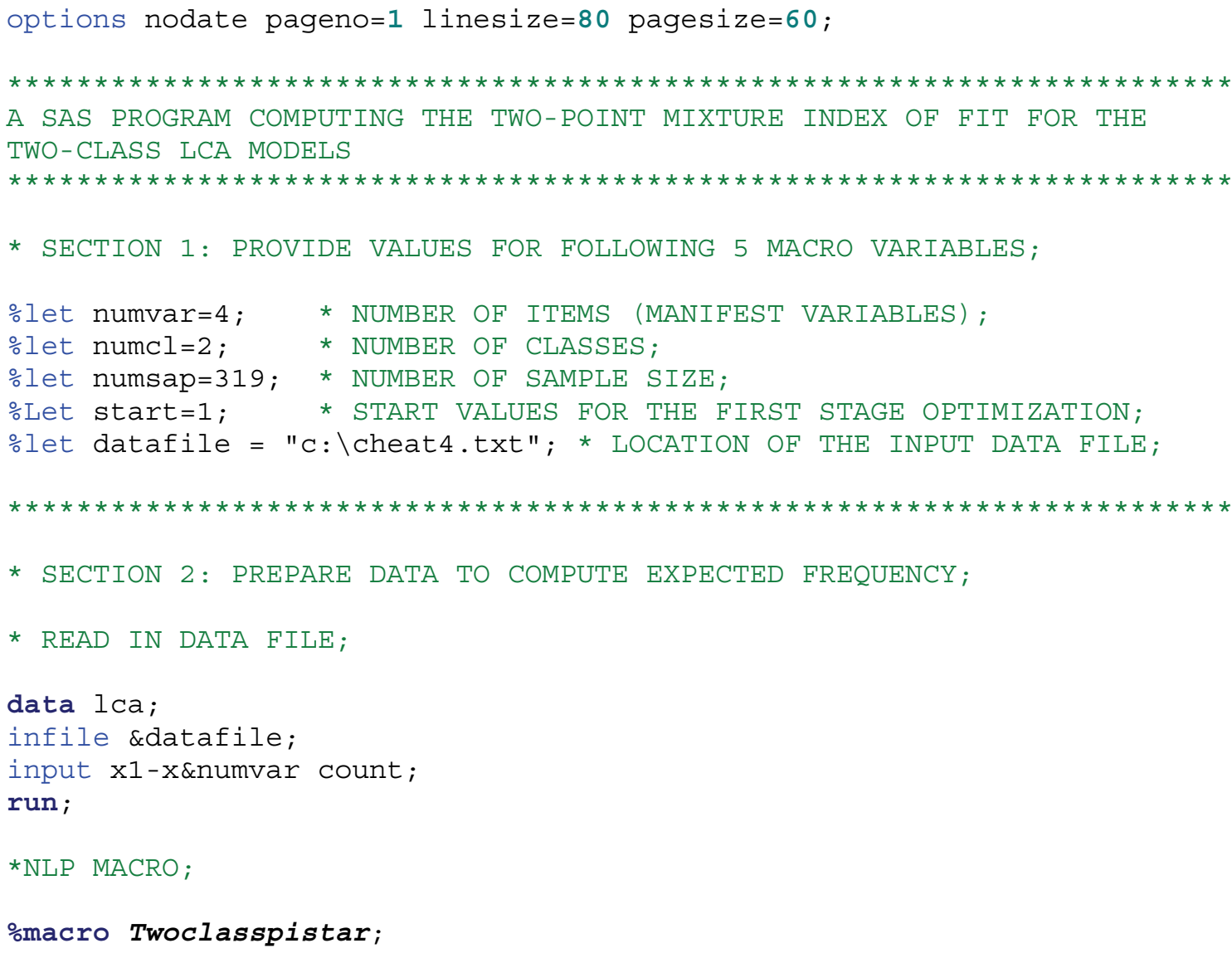




\section{ZHANG \& DAYTON}

Appendix: The SAS Program to Compute Pi-star with the Cheat4 Data (continued)

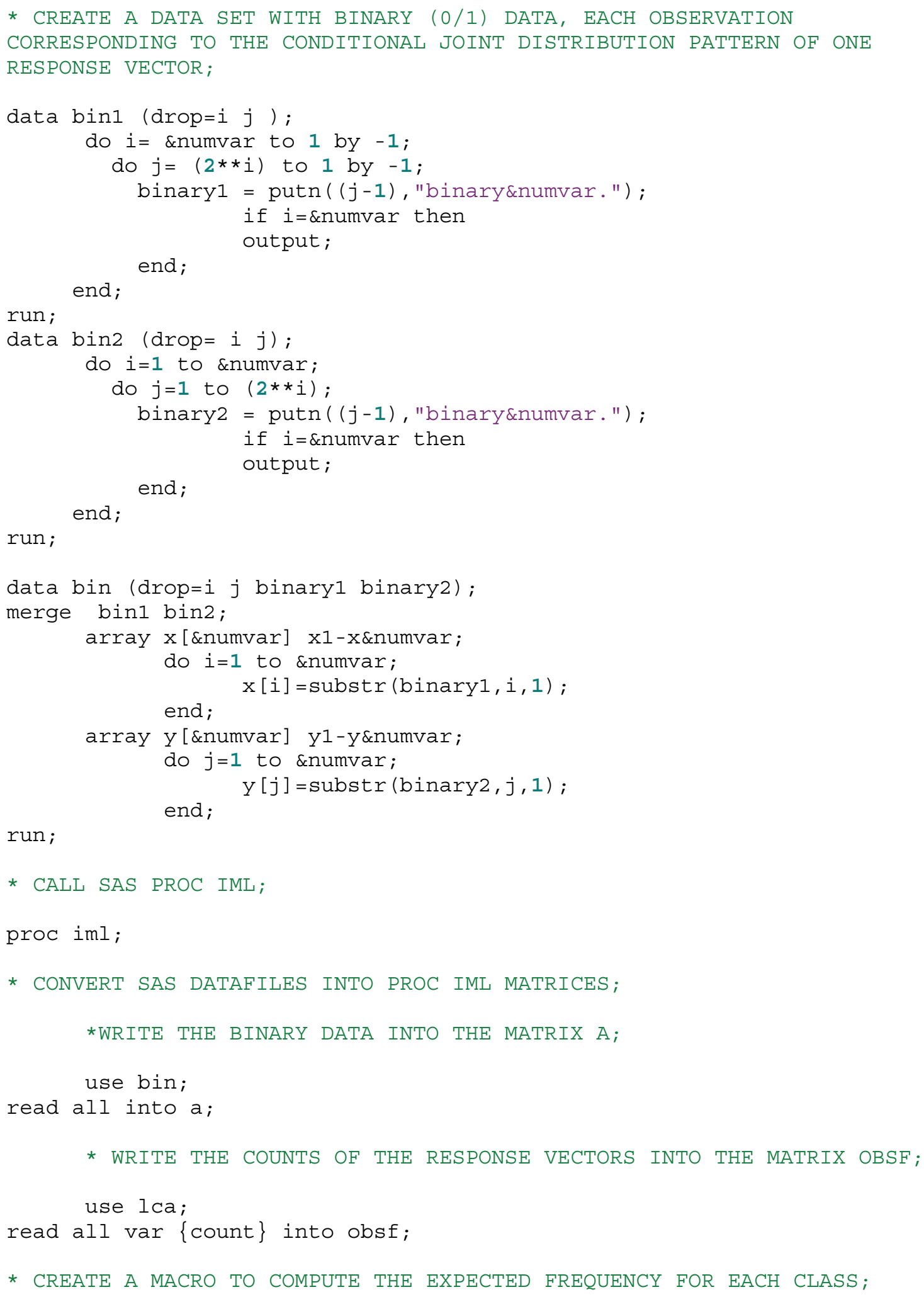




\section{PI-LCA SAS PROGRAM FOR TWO-CLASS LCA DICHOTOMOUS VARIABLE MODELS}

Appendix: The SAS Program to Compute Pi-star with the Cheat4 Data (continued)

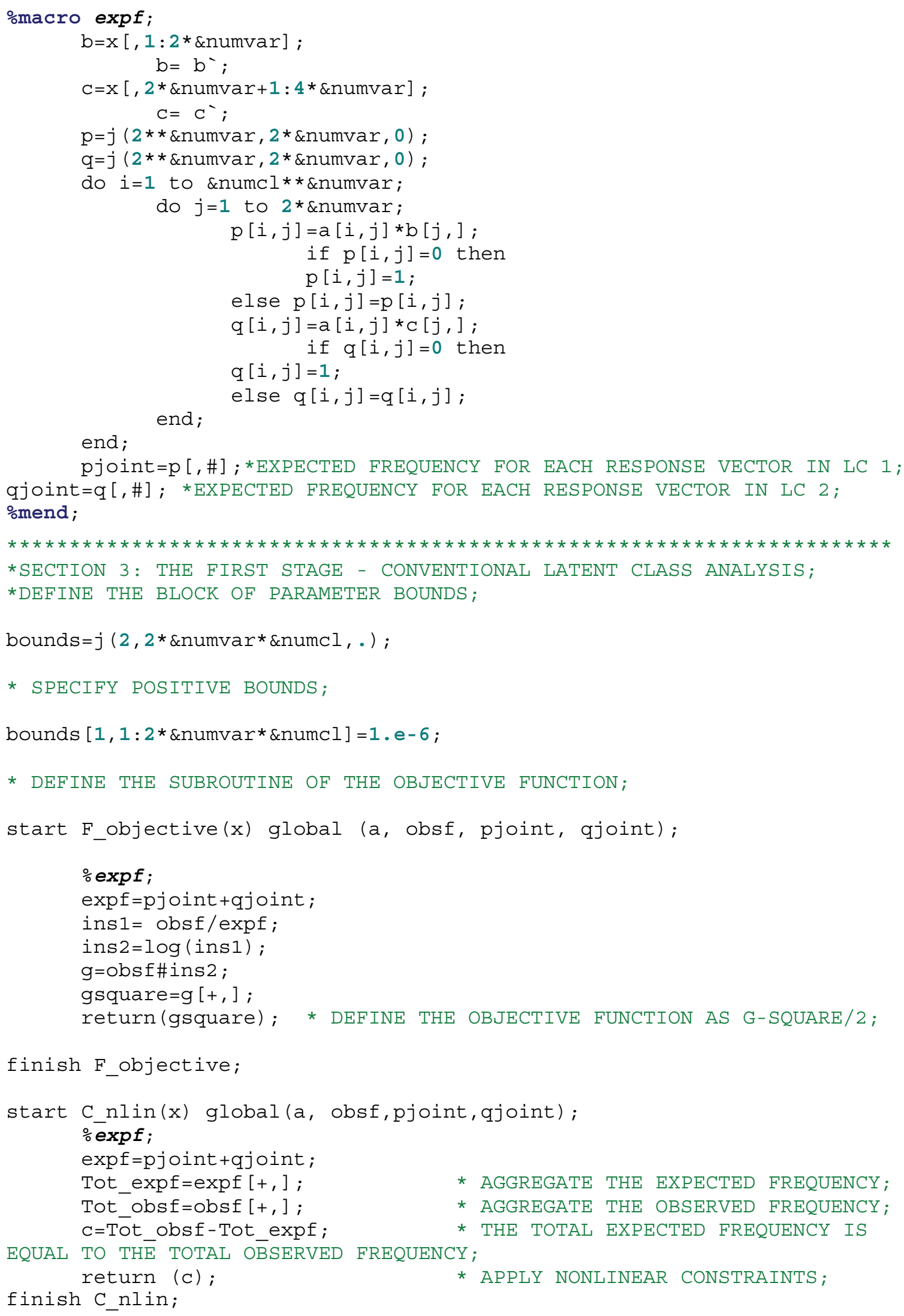




\section{ZHANG \& DAYTON}

\section{Appendix: The SAS Program to Compute Pi-star with the Cheat4 Data (continued)}

* NLP PROCEDURE;

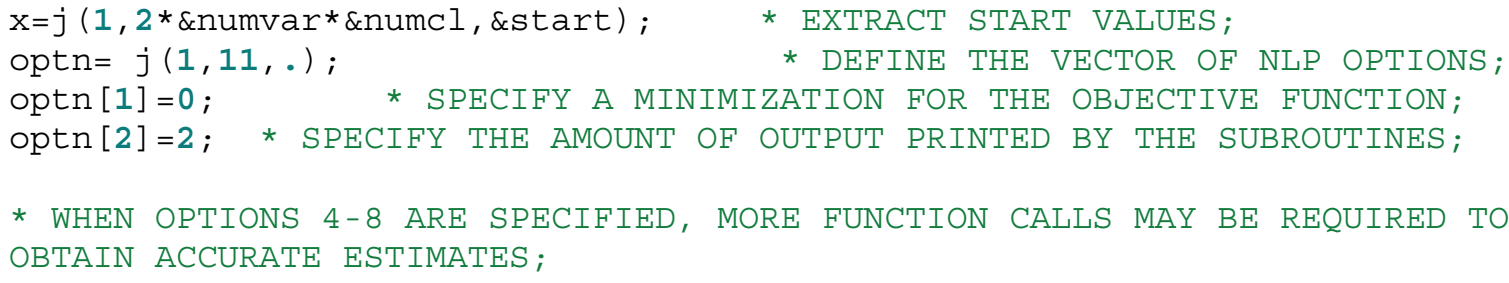




\section{PI-LCA SAS PROGRAM FOR TWO-CLASS LCA DICHOTOMOUS VARIABLE MODELS}

Appendix: The SAS Program to Compute Pi-star with the Cheat4 Data (continued)

* CREAte a marco to cOMPUte conditional probabilities;

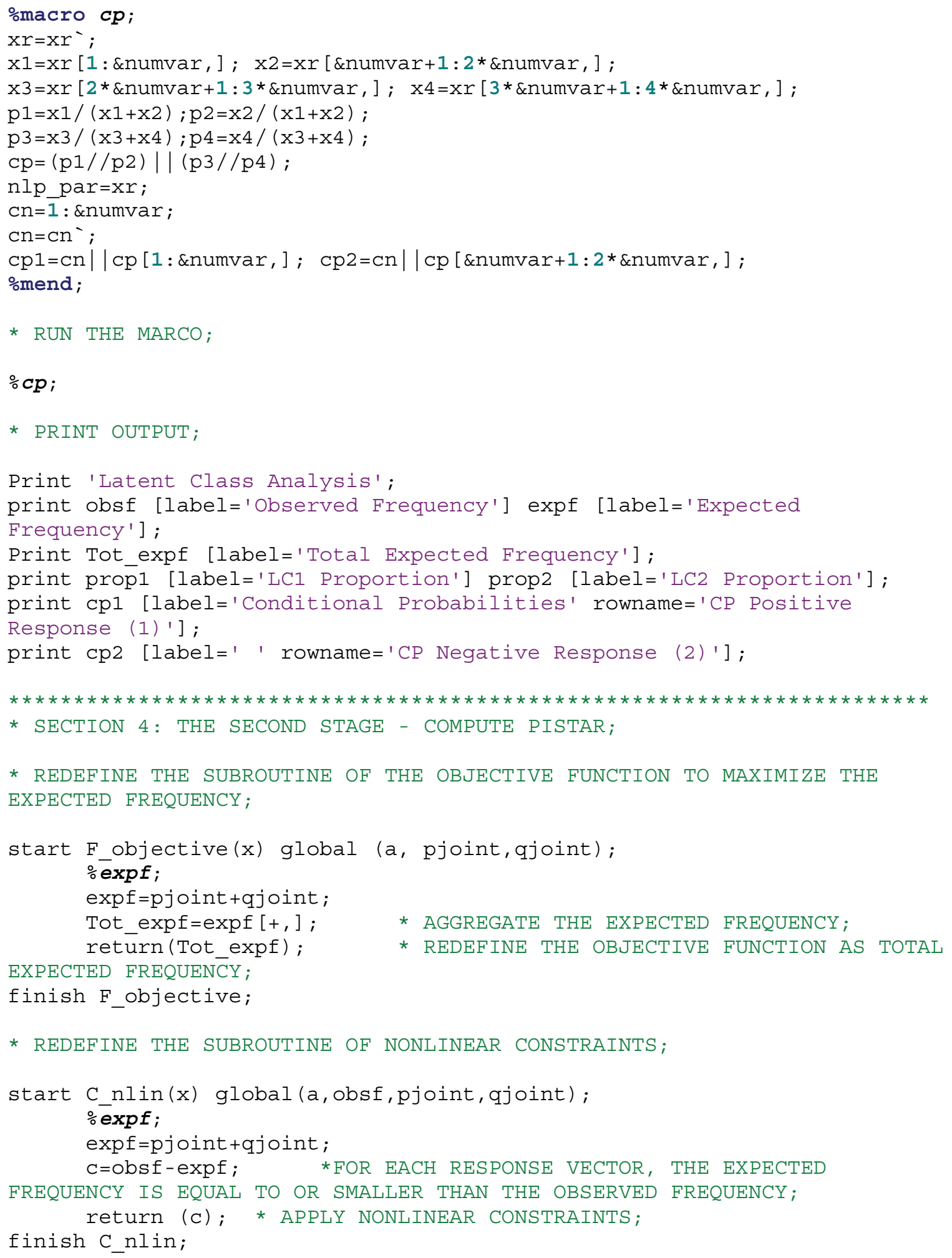




\section{ZHANG \& DAYTON}

Appendix: The SAS Program to Compute Pi-star with the Cheat4 Data (continued)

* CALL NLP PROCEDURE;

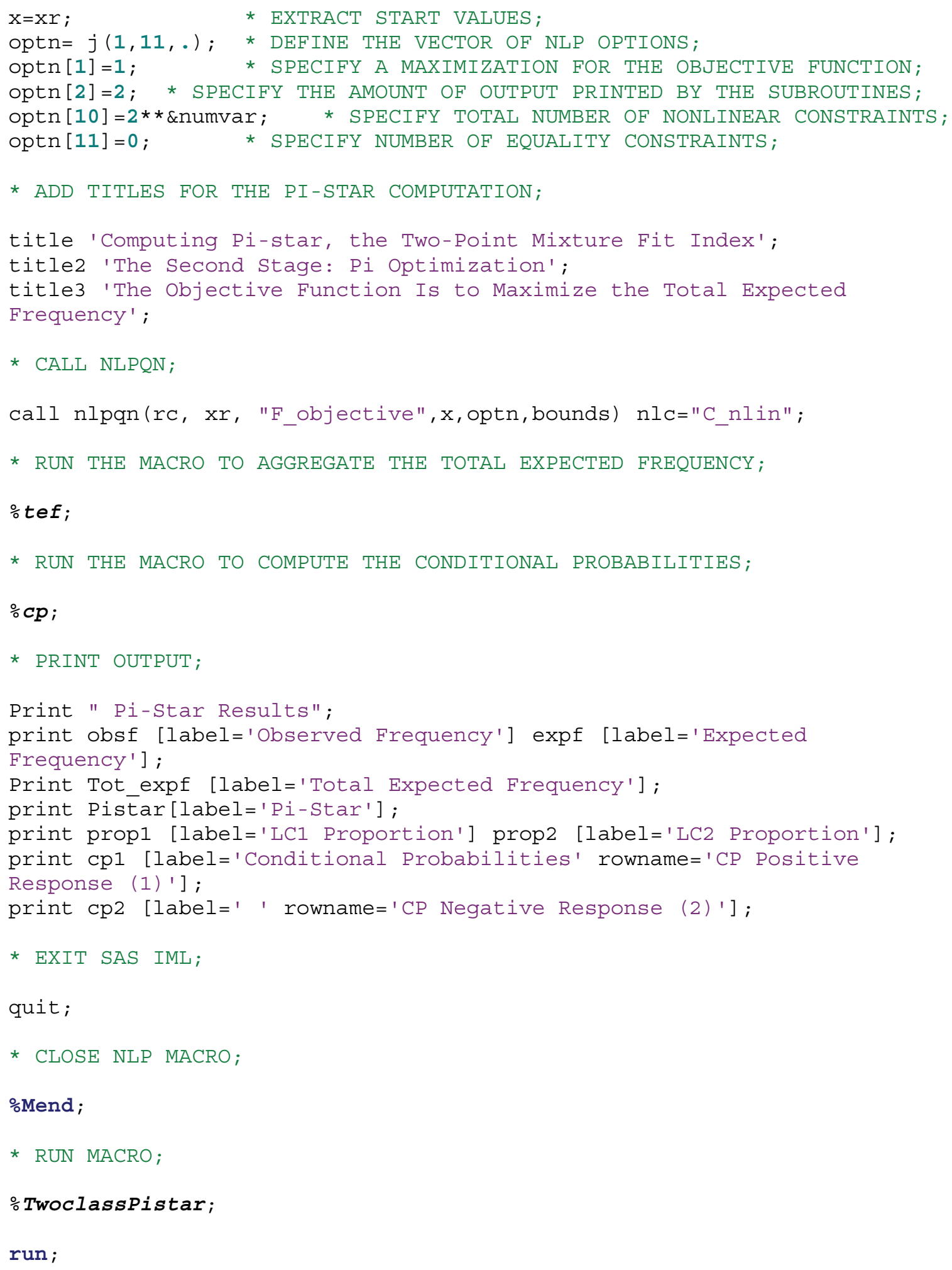

